\title{
Togitare
}

\section{CONSTRUÇÃO E VALIDAÇÃO DE INSTRUMENTO DE CONSULTA DE ENFERMAGEM PARA PESSOAS COM HEMOFILIA}

\author{
lara Alves Feitoza de Andrade ${ }^{1}$ (1) \\ Tânia Maria Rocha Guimarães ${ }^{2}$ (1) \\ Íris Maciel Costa ${ }^{1}$ (1) \\ Neuza Cavalcanti de Morais Costa ${ }^{1}$ (1) \\ Ricardo Mesquita Camelo ${ }^{3}$ (1) \\ Fábia Maria de Lima²
}

\section{RESUMO}

Objetivo: construir e validar um instrumento de consulta de enfermagem para pessoas com hemofilia.

Método: estudo metodológico realizado de fevereiro de 2017 a fevereiro de 2018, em um serviço referência de hematologia do Nordeste do Brasil. Utilizou-se a técnica Delphi para validação, por três grupos de enfermeiras juízas ( $n=29)$ : Especialistas em hemofilia ( $n=n o v e)$ de nove hemocentros do país; Residência em Hematologia ( $n=0$ ito); Enfermeiras do Serviço $(n=12)$. A concordância entre as juízas foi verificada por escala Likert e teste exato de Fisher.

Resultados: 89,6\% não apresentaram dificuldade para compreender o instrumento. Quanto ao grau de relevância, as características 'credibilidade' e cientificidade' apresentaram maiores percentuais de extremamente relevante (90\%). O teste exato de Fisher foi significativo no grau de satisfação de 'clareza das afirmações' $(p<0,05)$.

Conclusão: o instrumento foi considerado válido, proporcionando autonomia, apoio técnico e respaldo ético ao enfermeiro, contribuindo na melhoria da qualidade da assistência.

DESCRITORES: Enfermagem no Consultório; Estudo de Validação; Hemofilia A; Hemofilia B; Protocolo.

\section{CONSTRUCCIÓN Y VALIDACIÓN DE UN INSTRUMENTO DE CONSULTA DE ENFERMERÍA PARA PERSONAS CON HEMOFILIA}

\section{RESUMEN:}

Objetivo: construir y validar un instrumento de consulta de enfermería para personas con hemofilia. Método: estudio metodológico realizado de febrero de 2017 a febrero de 2018, en un servicio de referencia de hematología del Nordeste de Brasil. Para la validación se utilizó la técnica Delphi, por parte de tres grupos de enfermeras jueces $(n=29)$ : Especialistas en hemofilia ( $n=$ nueve) de nove hemocentros del país; Residencia en Hematología ( $n=0$ cho); Enfermeras del Servicio $(n=12)$. La concordancia entre las jueces se verificó mediante la escala Likert y la prueba exacto de Fisher. Resultados: 89,6\% não apresentaram dificuldade para compreender o instrumento. Quanto ao grau de relevância, as características 'credibilidade' e 'cientificidade' apresentaram maiores percentuais de extremamente relevante (90\%). O teste exato de Fisher foi significativo no grau de satisfação de 'clareza das afirmações' $(p<0,05)$. Conclusão: o instrumento foi considerado válido, proporcionando autonomia, apoio técnico e respaldo ético ao enfermeiro, contribuindo na melhoria da qualidade da assistência.

DESCRITORES: Enfermagem no Consultório; Estudo de Validação; Hemofilia A; Hemofilia B; Protocolo. 
A hemofilia é uma doença hemorrágica, genética, rara, com herança recessiva ligada ao cromossomo X. É caracterizada pela deficiência ou anormalidade da atividade coagulante do fator VIII (hemofilia A) ou do fator IX (hemofilia B). Do ponto de vista clínico, as hemofilias $A$ e $B$ são semelhantes, apresentando quadros hemorrágicos dependendo dos níveis plasmáticos do fator deficiente. As hemofilias podem ser classificadas como grave, quando o nível de fator circulante é menor que $1 \%$; moderada, entre $1 \%$ e $5 \%$ e; leve, de $5 \%$ a $40 \%{ }^{(1-2)}$.

Em 2017, o Brasil ( $n=12.432)$ tinha a quarta maior população mundial de Pessoas com Hemofilia $(\mathrm{PcH})$, ficando atrás da Índia $(n=18.966)$, Estados Unidos $(n=17.750)$ e China $(n=14.390)$, sendo a doença de maior prevalência entre as coagulopatias hereditárias ${ }^{(3)}$.

A consulta de enfermagem (CE) é parte fundamental do cuidado a $\mathrm{PcH}$, sendo considerada uma estratégia tecnológica importante e resolutiva, que oferece inúmeras vantagens no cuidado prestado(4-5). Neste contexto, o enfermeiro é um dos principais protagonistas do cuidado, porque realiza educação em saúde do paciente e familiares s $^{(1,4-6)}$, faz o treinamento para autoinfusão do fator em domicílio(7-9), monitora o progresso do tratamento, melhorando a qualidade e a segurança, incluindo a adesão ao tratamento( ${ }^{(9-10)}$.

Para garantir a confiabilidade à assistência de enfermagem através de procedimentos seguros, baseados em evidências científicas, é imprescindível a construção de protocolos. Há princípios estabelecidos para construção e validação de protocolos de assistência, como a definição clara do foco, da população a que se destinam, quem é o executor das ações, qual a estratégia de revisão da literatura, análise das evidências utilizadas, a forma de validação pelos pares, estratégias de implementação e a construção dos resultados esperados ${ }^{(11-12)}$.

A validade de conteúdo é a determinação da representatividade de itens que expressam um conteúdo, baseada no julgamento de especialistas em uma área específica, determinando se o conteúdo de um instrumento de medida explora, de maneira efetiva, os quesitos para mensuração de um determinado fenômeno a ser investigado(12).

Este artigo tem como objetivo descrever a construção e a validação de conteúdo de um instrumento de consulta de enfermagem para $\mathrm{PcH}$, em um serviço referência de hematologia do Nordeste brasileiro. A escassez de instrumentos de CE para pacientes com coagulopatias, e a grande complexidade de informações necessárias para a consulta, de forma a garantir que nenhum aspecto seja negligenciado, justificam a importância deste estudo.

\section{MÉTODO}

Estudo metodológico realizado no ambulatório de coagulopatias hereditárias de um serviço referência de hematologia do Nordeste brasileiro, situado em Recife-PE. A coleta de dados foi realizada de fevereiro de 2017 a fevereiro de 2018.

O referencial teórico seguido no estudo(13) sugere um roteiro de quatro etapas para guiar o processo de construção e validação de instrumentos: 1. Planejamento (objetivo, população alvo, itens do instrumento); 2. Construção (conteúdo, avaliação da validade de conteúdo por um painel de especialistas); 3. Validaçãa (aplicação do teste em um número significativo de juízes para validade de conteúdo e de aparếncia). 4. Avaliação final do instrumento (aplicação da versão piloto em um grupo experimental apropriado). 


\section{$1^{\text {a Etapa: Planejamento }}$}

Nesta etapa foram identificados indicadores empíricos (variáveis sociodemográficas e clínicas) e das necessidades humanas afetadas de $\mathrm{PcH}$, através da observação randomizada das CE no ambulatório do serviço. Realizou-se também levantamento bibliográfico com os descritores "estudos de validação" combinados com "enfermagem no consultório", "hemofilia $A$ " e "hemofilia B" utilizando o operador booleano 'AND', nas bases de dados BDENF, BIREME/BVS, LILACS, MEDLINE/PubMed, Ministério da Saúde do Brasil, SCIELO e World Federation of Hemophilia.

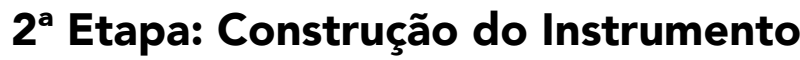

A primeira versão do instrumento foi construída segundo modelo do Hemovida WebCoagulopatias do Ministério da Saúde do Brasil(14), porque o levantamento bibliográfico realizado não encontrou artigos sobre instrumento de CE para hemofilia. Desta forma, o instrumento foi elaborado por duas enfermeiras do serviço com 10 anos dedicados exclusivamente a $\mathrm{PcH}$, com média de 110 atendimentos/mês. Posteriormente, o instrumento foi analisado pela técnica do grupo focal, por uma equipe multiprofissional de 12 especialistas do ambulatório (um assistente social, duas enfermeiras, duas psicólogas, um fisioterapeuta, um dentista, uma farmacêutica e quatro médicas).

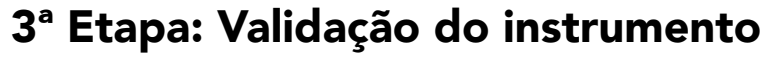

Nesta etapa, o instrumento foi avaliado por enfermeiras juízas através da técnica Delphi ${ }^{(15)}$, que consiste em avaliar um determinado tópico por meio do julgamento de especialistas no assunto, baseando-se na opinião convergente dos avaliadores, enfatizando a necessidade de consenso do grupo. Também utilizou-se a validação de aparência, mesmo sendo considerada uma técnica subjetiva ${ }^{(12)}$, entretanto consideramos importante devido à avaliação ter sido realizado por especialistas de nove estados brasileiros, propiciando uma maior confiabilidade ao instrumento.

O tamanho da amostra das juízas foi definido pela fórmula $\mathbf{n}=\mathbf{Z} \boldsymbol{\alpha}^{2} \cdot \mathbf{P} .(1-\mathbf{P}) / \mathbf{d}^{2}$, onde $\mathbf{Z} \boldsymbol{\alpha}$ refere-se ao nível de confiança (convencionou-se 95\%), $\mathbf{P}$ é a proporção de indivíduos que concordam com a pertinência dos conceitos (convencionou-se $85 \%$ ), e d é a diferença de proporção considerada aceitável (convencionou-se 15\%)(16). O cálculo final foi determinado por $\mathbf{n}=\mathbf{1}, \mathbf{9 5}^{\mathbf{2}} \cdot \mathbf{0}, \mathbf{8 5} \cdot \mathbf{0}, \mathbf{1 5} / \mathbf{0}, \mathbf{1 5}^{\mathbf{2}}$ e com isso, obteve-se uma amostra de 22 juízas, sendo acrescentado $20 \%$ para perdas ou recusas, totalizando 26 juízas.

Os critérios de seleção das juízas foram: 1. Especialistas em Hemofilia ( $E H, n=n o v e)$ de nove hemocentros do país (Campinas, Ceará, João Pessoa, Maceió, Manaus, Natal, Minas Gerais, Recife e Rio de Janeiro) definida por meio da amostragem de bola de neve ${ }^{(17)}$, na qual o participante selecionado indica outros participantes, tratando-se de uma amostra por conveniência, sendo enviados por endereço eletrônico a carta convite e a ficha de avaliação; 2. Enfermeiros com Residência em Hematologia (ER, $n=0$ ito); 3. Enfermeiros do Serviço (ES, $n=12)$, todas com experiência de mais de três anos no atendimento a $\mathrm{PcH}$. Adotou-se como critérios de exclusão enfermeiras afastadas por licença médica, prêmio e férias.

As juízas foram convidadas a participar do estudo, orientadas dos objetivos e assinaram o Termo de Consentimento Livre e Esclarecido. Todas as enfermeiras aceitaram participar do estudo e o grupo EH enviou as respostas por meio de endereço eletrônico. Conseguiu-se obter uma amostra de 29 juízas, conferindo maior confiança à validação efetuada, atendendo ao quantitativo recomendado de uma amostra de 25 a 50 especialistas para realizar a validação(15).

A concordância entre as juízas foi verificada pela escala likert de quatro pontos ${ }^{(18)} \mathrm{e}$ 
pelo teste exato de Fisher, aplicado para comparar a concordância entre as especialistas, para verificar a homogeneidade da aplicação do instrumento. A ficha de avaliação analisou: A. Grau de dificuldade de preenchimento (dificuldade completa-1, dificuldade moderada-2, dificuldade leve-3, sem dificuldade-4); B. Grau de relevância das características: objetividade, simplicidade, clareza, pertinência, precisão, credibilidade e cientificidade (não relevante-1, pouco relevante-2, relevante-3, extremamente relevante-4); C. Grau de satisfação sobre aparência do instrumento: apresentação, clareza das questões, facilidade de leitura, interpretação e representatividade (sem impacto-1, insuficiente-2, satisfatório-3, ótimo-4).

A pontuação de cada item foi tabulada com uma contagem simples do número de respostas para cada critério de avaliação e a porcentagem de juízas que concordaram com o conteúdo do instrumento. Os escores um e dois foram considerados indicativos de baixa qualidade, e os escores três e quatro foram considerados indicativos de boa qualidade. Foram considerados válidos os itens com no mínimo $85 \%$ de concordância.

Para análise estatística dos dados, foi construído um banco na planilha Microsoft Excel 2013, que foi exportada para o software SPSS-18, onde foi realizada a análise. Todas as conclusões foram tiradas considerando o nível de significância de $5 \%$.

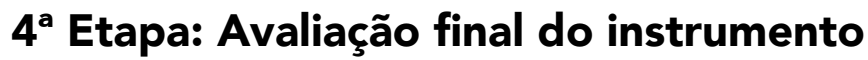

Esta etapa constou da realização do teste piloto da versão final do instrumento a 30 $\mathrm{PcH}$ adultas, que compareceram às CE no ambulatório da instituição, conforme critérios estabelecidos na literatura, que definem uma amostra de 30 a 40 pessoas $^{(19)}$.

O estudo foi realizado respeitando-se a resolução 466/2012 do Conselho Nacional da Saúde do Ministério da Saúde, sendo aprovado pelo Comitê de Ética em Pesquisa da instituição pesquisada sob parecer número 1.863.411.

\section{RESULTADOS}

Quanto à caraterização das juízas, todas eram do sexo feminino. $O$ grupo ER era mais jovem, apresentando média de idade $(30 \pm 6,4)$, EH $(47 \pm 9,8)$ e ES $(51 \pm 12)$. No grupo $\mathrm{EH}$, verificou-se uma titulação de doutorado $(11,1 \%)$, duas de mestrado $(22,2 \%)$ e três proficiências em hematologia (33,3\%); no ER todas tinham concluído a residência de enfermagem em hematologia há menos de cinco anos; e no ES seis tinham titulação de mestrado (50\%) em áreas diferentes de hematologia.

Considerando as sugestões dadas pelas juízas (Quadro 1), verificou-se um total de 19 sugestões das quais $12(63,2 \%)$ foram aceitas pelos pesquisadores. Observou-se que o grupo EH apresentou mais sugestões $11(57,9 \%)$, seguido pelo ES cinco $(26,3 \%)$ e ER três $(15,8 \%)$.

Quadro 1 - Lista de sugestões das enfermeiras juízas. Recife, PE, Brasil, 2018 (continua)

\begin{tabular}{|c|c|c|}
\hline Itens & Sugestão dos Juízes & $\begin{array}{l}\text { Aceitação dos } \\
\text { Pesquisadores }\end{array}$ \\
\hline \multirow{2}{*}{ Dados gerais } & 1. Ampliar o instrumento para todas as coagulopatias hereditárias & Não \\
\hline & 2. Especificar todos os medicamentos & Não \\
\hline
\end{tabular}




\begin{tabular}{|c|c|c|}
\hline & 3. Inserir número de filhos & Sim \\
\hline & 4. Retirar o item sobre glicemia & Não \\
\hline \multirow{2}{*}{ Hábitos } & 5. Inserir número de cigarros/dia & Sim \\
\hline & 6. Transportar para atividades sociais & Não \\
\hline Alergias & 7. Compor bloco 1 & Sim \\
\hline \multirow{4}{*}{$\begin{array}{l}\text { Pesquisa de } \\
\text { Inibidor }\end{array}$} & 8. $\quad$ Trocar ordem com item 11 & Não \\
\hline & 9. Acrescentar treinamento de autoinfusão & Sim \\
\hline & 10. Acrescentar data do último treinamento & Sim \\
\hline & 11. Esclarecer os títulos: primeiro título, pico histórico, título geral & Sim \\
\hline Mobilidade & $\begin{array}{l}\text { 12. Mudar o termo "deambula com auxílio" para "deambula com } \\
\text { dificuldade" }\end{array}$ & Não \\
\hline Sorologias & 13. Inserir de imunização & Sim \\
\hline \multirow{3}{*}{$\begin{array}{l}\text { Atividades } \\
\text { Sociais }\end{array}$} & 14. Inserir atividade sexual & Sim \\
\hline & 15. Inserir atividades esportivas & Sim \\
\hline & 16. Inserir planejamento familiar & Sim \\
\hline $\begin{array}{l}\text { Conferência do } \\
\text { diário de infusão }\end{array}$ & 17. Inserir descarte correto do material & Sim \\
\hline $\begin{array}{l}\text { Diagnósticos de } \\
\text { Enfermagem }\end{array}$ & 18. Trocar ordem com item 17 & Não \\
\hline Imunotolerância & 19. Inserir opção de "presença de inibidor" & Sim \\
\hline
\end{tabular}

Fonte: Autores (2018)

Em relação ao grau de dificuldade das juízas para compreender o instrumento, constatou-se que a maioria não apresentou nenhuma dificuldade $15(51,7 \%)$, seguida de dificuldade leve $11(37,9 \%)$ e dificuldade moderada três $(10,4 \%)$. O teste exato de Fisher $(p=0,358)$ não foi significativo, indicando que o grau de dificuldade é semelhante entre os grupos analisados.

A análise das características do instrumento quanto à estrutura, estética e conteúdo mostrou um padrão similar de resposta, apresentando uma única divergência no item 'clareza de informação', onde o grupo EH apresentou menor percentual de 'ótimo'; e sugeriu que o instrumento fosse ampliado para todas as coagulopatias hereditárias. Entretanto, optou-se por manter o instrumento sem mudança, porque seria necessário inserir vários itens referentes à saúde da mulher, para atender o perfil feminino das demais coagulopatias hereditárias.

O instrumento foi também considerado longo pelo grupo $\mathrm{EH}$, apesar de todas as enfermeiras terem sido orientadas que seria implantado no formato eletrônico, e que o preenchimento completo ocorreria somente na primeira consulta, e posteriormente, os dados seriam apenas atualizados.

A relevância das características avaliadas no instrumento está descrita na Tabela 1. Verificou-se que 'credibilidade' e 'cientificidade' apresentaram os maiores percentuais de "extremamente relevante". Entretanto, excluindo-se essas características, observou-se maior percentual de "extremamente relevante" nos grupos EH (precisão), ER (objetividade e pertinência) e ES (clareza) do instrumento. O teste de comparação não foi significativo em todas as características $(p>0,05)$. 
Tabela 1 - Distribuição do grau de relevância das características do instrumento avaliadas pelas enfermeiras juízas ( $n=29)$, segundo a formação/atuação profissional. Recife, PE, Brasil, 2018

\begin{tabular}{|c|c|c|c|c|c|}
\hline \multirow[t]{2}{*}{ Característica avaliada } & \multirow{2}{*}{$\begin{array}{l}\text { Total } \\
\text { n (\%) }\end{array}$} & \multicolumn{3}{|c|}{ Grupo avaliado } & \multirow{2}{*}{$\begin{array}{c}\text { p-valor } \\
\left({ }^{*}\right)\end{array}$} \\
\hline & & $\begin{array}{c}\text { EH } \\
n(\%) \\
(n=9)\end{array}$ & $\begin{array}{c}\text { ER } \\
n(\%) \\
(n=8)\end{array}$ & $\begin{array}{c}E S \\
n(\%) \\
(n=12)\end{array}$ & \\
\hline \multicolumn{6}{|l|}{ Clareza } \\
\hline Relevante & $8(28)$ & $3(33)$ & $3(38)$ & $2(17)$ & \multirow{2}{*}{0,582} \\
\hline Extremamente relevante & $21(72)$ & $6(67)$ & $5(62)$ & $10(83)$ & \\
\hline \multicolumn{6}{|l|}{ Pertinência } \\
\hline Relevante & 9(31) & $3(33)$ & $1(12)$ & $5(42)$ & \multirow{2}{*}{0,468} \\
\hline Extremamente relevante & $20(69)$ & $6(67)$ & $7(88)$ & $7(58)$ & \\
\hline \multicolumn{6}{|l|}{ Precisão } \\
\hline Relevante & $7(24)$ & $1(11)$ & $3(38)$ & $3(25)$ & \multirow{2}{*}{0,407} \\
\hline Extremamente relevante & $22(76)$ & $8(89)$ & $5(62)$ & $9(75)$ & \\
\hline \multicolumn{6}{|l|}{ Credibilidade } \\
\hline Relevante & $3(10)$ & $1(11)$ & $1(12)$ & $1(8)$ & \multirow{2}{*}{1} \\
\hline Extremamente relevante & $26(90)$ & $8(89)$ & $7(88)$ & $11(92)$ & \\
\hline \multicolumn{6}{|l|}{ Cientificidade } \\
\hline Relevante & $3(10)$ & $1(11)$ & $1(12)$ & $1(8)$ & \multirow{2}{*}{1} \\
\hline Extremamente relevante & $26(90)$ & $8(89)$ & $7(88)$ & $11(92)$ & \\
\hline
\end{tabular}

Legenda: EH - Enfermeira Especialista em Hemofilia; ER - Enfermeira com Residência em Hematologia e Hemoterapia; ES Enfermeira do Serviço; $\left(^{*}\right)$ Teste Exato de Fisher $(p<0,05$ existe diferença da opinião entre os grupos de especialistas).

Fonte: Autores (2018)

Em relação ao grau de satisfação das juízas sobre a aparência do instrumento (Tabela 2), verificou-se que a característica 'representatividade' apresentou os maiores percentuais de ótimo. O grupo ER considerou todas as características analisadas como ótimas, apresentando maiores percentuais de satisfação. O grupo EH apresentou menor percentual de ótimo em relação à característica 'clareza das afirmações', resultando em um teste exato de Fisher significativo $(p<0,05)$, indicando que existe diferença da opinião entre os grupos.

Tabela 2 - Distribuição do grau de satisfação das enfermeiras juízas sobre os itens de aparência do instrumento, segundo a formação/atuação profissional. Recife, PE, Brasil, 2018 (continua)

\begin{tabular}{lcccc} 
Característica avaliada & Total & \multicolumn{3}{c}{ Grupo avaliado } \\
\cline { 3 - 5 } & $n(\%)$ & p-valor \\
& & $\begin{array}{c}\text { EH } \\
(\%) \\
(n=9)\end{array}$ & $\begin{array}{c}n(\%) \\
(n=8)\end{array}$ & $\begin{array}{c}n(\%) \\
(n=12)\end{array}$ \\
\hline
\end{tabular}

Apresentação 


\begin{tabular}{|c|c|c|c|c|c|}
\hline Satisfatória & $7(24)$ & $2(22)$ & $1(12)$ & $4(33)$ & \multirow{2}{*}{0,664} \\
\hline Ótima & $22(76)$ & $7(78)$ & $7(88)$ & $8(67)$ & \\
\hline \multicolumn{6}{|c|}{ Clareza das afirmações } \\
\hline Insuficiente & $1(3)$ & 0 & $1(12)$ & 0 & \multirow{3}{*}{0,035} \\
\hline Satisfatória & $8(28)$ & $5(56)$ & 0 & $3(25)$ & \\
\hline Ótima & $20(69)$ & $4(44)$ & $7(88)$ & $9(75)$ & \\
\hline \multicolumn{6}{|c|}{ Facilidade de leitura } \\
\hline Insuficiente & $2(7)$ & 0 & $1(12)$ & $1(8)$ & \multirow{3}{*}{0,434} \\
\hline Satisfatória & $5(17)$ & $3(33)$ & 0 & $2(17)$ & \\
\hline Ótima & $22(76)$ & $6(67)$ & $7(88)$ & $9(75)$ & \\
\hline \multicolumn{6}{|c|}{ Representatividade } \\
\hline Insuficiente & $1(3)$ & $1(11)$ & 0 & 0 & \multirow{3}{*}{0,338} \\
\hline Satisfatória & $4(14)$ & 0 & $1(12)$ & $3(25)$ & \\
\hline Ótima & $24(83)$ & $8(89)$ & $7(88)$ & $9(75)$ & \\
\hline
\end{tabular}

Legenda: EH - Enfermeira Especialista em Hemofilia; ER - Enfermeira com Residência em Hematologia e Hemoterapia; ES Enfermeira do Serviço; $\left(^{\star}\right)$ Teste Exato de Fisher ( $p<0,05$ existe diferença da opinião entre os grupos de especialistas).

Fonte: Autores (2018)

A versão final do instrumento foi composta por duas partes, a primeira (Figura 1) com dados gerais com 19 categorias, e a segunda (Figura 2) com dados de Imunotolerância. 


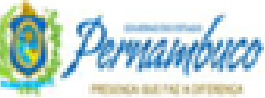

\section{HEMOPE}

\section{INSTRUMENTO DE CONSULTA DE ENFERMAGEM PARA PESSOA COM HEMOFILIA -HEMOPE}

Pronanirio Noene Sexo Data de Nascimento: Grau de Instruçlio:

1. DADOS GERAIS: Código HWC ___ Tipo:_ Gravidade:__ Peso___ Alnara PA. Glicemia Medicaçio de uso contimo $\mathrm{ABO}(\mathrm{RH}(\mathrm{C})$

2. HABrroS (Se Sim, informar $\mathrm{A}=$ Atual ou $\mathrm{P}=\mathrm{Passado}$ )

Tabagista: $\operatorname{Sim}$ ( ) N3o ( ) Quantos cigarros ao dia? __Etilista: Sim ( ) N3lo ( ) Drogas ilicitas: $\operatorname{Sim}$ ( ) N3lo( )

3. ALERGLAS: Sim ( ) Näo ( ) Qual?

4. TRATAMENTO: Tipo:___ Produto:__ Esquema Administrado por: Paciente ( ) Cuidador ( ) Prof de Saude ( ) Local: Domiciliar ( ) Hospitalar ( ) CTH( ) Acesso Venoso Periférico: Ótimo ( ) Bom( ) Ruim( ) Acesso Venoso Central: Sim ( ) N3o ( ) Dispositivo: Data da Inserçio: Data da Retirada: Manipulado por:

Motrvo

5. PESQUTSA DE NIBIDOR: Presenç: Sim ( ) Nio( ) Úthima Dosagem:

Data da Dosagem:

Negativaçio espontänea ( ) Negativaçảo Pós-Imunotolerajecia ( ) Em Espera ( ) Em IT( ) Failha ( ) $\overline{R e c i d i v a ~(~) ~}$ Contraindicado ( ) Recusa ( ) Abandono ( ) Pico Histórico:

Data da primeira exposiçio a Fator: ___ _ _ Qual idade?

Histónico Familiar de Inibidor: Sim ( $)$ Năo( ) Quem?

Usou: Fator $>50 \mathrm{UI} \mathrm{kg}$ /dose/ > 5 Dias continuos? Sim ( ) N3o( Primeiro Tínlo: $\quad$ UB $/ \mathrm{mL}$. Data do $1^{\circ}$ Titulo Data do Pico:

6. TRENAMENTO DE INFUSÃo/AUTONFUSÃo (ANUAL) Data: Qual idade? Genotipagem:

Higienizaçào das mãos ( ) Preparo do Fator ( ) Infusajo ( ) Descarte ( ) Acondicionamento domiciliar ( )

7. EVENTOS HEMORRAGICOS, DEPOIS DA ÚLTIMA CONSULTA

$1^{*}$ Local Espontaineo ( ) Trauma ( ) Data

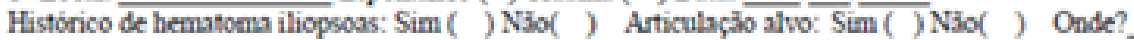
Quantidade de eventos bemorragicos em articulaçóes alvo nos ultimos 12 meses: Articulaçes Comprometidas: Sim ( ) Nio( ) Quais?

8. MOBIL.IDADE: Deambula: Sem Auxilio ( ) Com Auxilio( ) Qual: Cadeirante? Sim ( ) N3o ( )

9. PROCEDIMINTOS NVASTVOS: Radiosinoviontese: $\operatorname{Sim}($ ) N3io ( ) Data Histórico de cirurgias: Sim( ) Nio( ) Quais?

10.SOROLOGLAS POSIITVAS Data do tiltimo exame: Anti-HBC ( ) Anti-HCV ( ) HBsAg( ) Anti-HIV( ) Anti-HTLV( ) Chagas ( ) Anti-HBs ( ) VDRL () Tratamento em Serviç Especializado: Nuaca procurou atendimento ( ) Procurou Tratamento, mas abandonou ( ) Em Tratamento ( ) Em Farmacoterapia ( ) Alta com Laudo Medico ( ) Alta sem Laudo Médico ( )

11. MuNIZAÇ.̇̃o: Hepatite B: 1 Dose ( ) 2 Doses ( ) 3 Doses ( ) N3̈o Vacinado ( ) Hepatite A: 1 Dose ( ) 2 Doses ( ) Nio Vacinado ( ) Pneumococos: 1 Dose ( ) Nio Vacinado ( )

\section{ATIVTDADES SOCLAIS}

Atividade Laboral: $\operatorname{Sim}($ ) Nalo( ) Qual?

Exercicio Fisico: Sim ( ) Nảo( ) Qual?

Lazer: $\operatorname{Sim}($ ) $\mathrm{N3o}($ ) Qual?

Atividade Sexual: Ativa ( ) Nio Ativa ( ) Planj. Regrodutivo: Sim ( ) Năo( ) Qual? $\mathrm{N}^{*}$ Fillhos:

13. GRAU DE INDEPENDÊNCLA: Escore de Independencia Funcional em Hemofilia (FISH) - Avaliaçio Anual* Total do Escore (32 pontos). Pontuaçào: 1 - Incapaz de executar atividade ou necessita de assistencia completa para exerce-la. 2 Precisa de assistëncia parcial $e$ instrumentos ou ambientes modificados para executar atividade; 3 - Apto a executar atividade sem ajuda ou assisténcia, porém com ligeiro desconforto; 4 - Apto a executar qualquer atividade sem dificuldade alguma, como seus colegas sandiveis.
A - AUTOCUIDADOS: Alimenta-se e Amama-se:
B - TRANSFERENCIAS: Sentar-se e Levantar-se:
C-LOCOMOÇÃO: Padrío de Marcha: Tomar Banho:
Resultado: Data:
15. EM ACONPANHAMENTO MULTIPROFISSIONAL: Sim ( ) N3o( ) Qua?
16. CONFERENCIA E AVALLAĊ̃O DO DLARIO DE INFUSĀo
17. EXAMIE FISICO, OBSERVACCÓES E ORIENTACYOES;
18. DLAGNOSTICOS DE ENFERMLAGEM: Vestir-se: Subir e Descer Escadas (12 - 14 Degraus): ___ Correr

14 QUEIXAS

19. ENCAMIHAMIENTOS

Data

Nome do (a) Enfermeiro (a) / COREN

Figura 1 - Instrumento de Consulta de Enfermagem para Pessoa com Hemofilia. Recife, PE, Brasil, 2018 


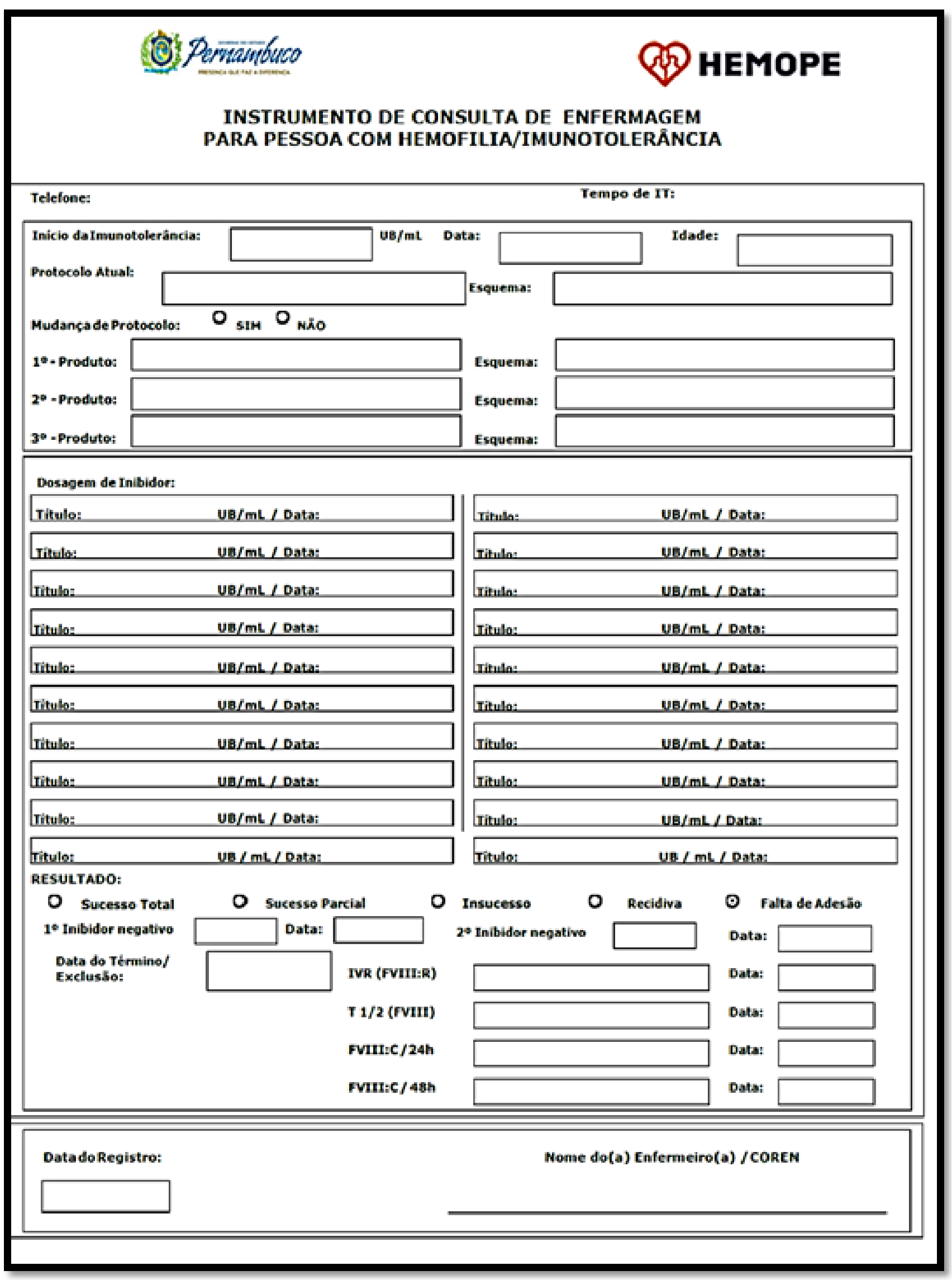

Figura 2 - Instrumento de Consulta de Enfermagem para Pessoa com Hemofilia/ Imunotolerância. Recife, PE, Brasil, 2018 
No teste piloto realizado no ambulatório com $30 \mathrm{PcH}$, verificou-se que as enfermeiras não tiveram dificuldades na aplicação do instrumento. A primeira consulta foi mais longa, em torno de 30 minutos; as consultas subsequentes, quando os dados foram apenas atualizados, levou de 10 a 15 minutos. A parte mais demorada foi a aplicação do Escore de Independência Funcional em Hemofilia (FISH), que deve ser preenchido semestralmente, e a avaliação da autoinfusão do fator de coagulação, que deve ser realizado anualmente.

\section{DISCUSSÃO}

A utilização de instrumentos de medida nas práticas de saúde cresce progressivamente. $\mathrm{Na}$ área da enfermagem, validar instrumentos que norteiem a prática é sinônimo de desenvolvimento de tecnologias de saúde para a profissão, uma vez que se torna possível direcionar os cuidados de enfermagem e melhorar a qualidade da assistência ${ }^{(20)}$.

Vantagens têm sido apontadas para o uso de instrumentos de assistência, tais como maior segurança aos usuários e profissionais, redução da variabilidade de ações de cuidado, melhora na qualificação dos profissionais para a tomada de decisão assistencial, facilidade para a incorporação de novas tecnologias, inovação do cuidado, uso mais racional dos recursos disponíveis e maior transparência e controle dos custos. Ainda como vantagens, protocolos facilitam o desenvolvimento de indicadores de processo e de resultados, a disseminação de conhecimento, a comunicação profissional e a coordenação do cuidado ${ }^{(11,19)}$.

Segundo recomendações de especialistas em coagulopatias hereditárias do Nordeste brasileiro sobre o papel da enfermeira na assistência de pessoas com hemofilia, descreve a importância do uso de instrumentos validados: "A consulta de enfermagem à pessoa com hemofilia deve ser realizada em todos os centros de tratamentos de hemofilia, por meio de instrumento padronizado e validado, aplicado por enfermeira com experiência em coagulopatias hereditárias"(21: e121).

As recomendações visam unificar a enfermagem na prática assistencial, garantindo sua liderança, seu papel na consulta, com autonomia para selecionar pacientes que são candidatos a tratamento de profilaxia, solicitar exames laboratoriais de acordo com protocolos internos e participar da decisão terapêutica(21).

Vários estudos ${ }^{(22-25)}$ demonstram que instrumentos válidos quanto ao seu conteúdo subsidiam o desenvolvimento na prática assistencial de enfermagem, e ao utilizar a técnica Delphi para alcançar o consenso entre especialistas, minimiza a influência direta, permite o acesso a participantes distantes e favorece o raciocínio pessoal e clínico.

A consulta de enfermagem à PcH está preconizada no Manual de hemofilia brasileiro(26) e nas Diretrizes para o Tratamento da Hemofilia da Federação Mundial de Hemofilia(1) como parte fundamental do atendimento ao paciente, sendo considerada uma estratégia tecnológica de cuidado em saúde importante e resolutiva, respaldada por lei, privativa do enfermeiro, e que oferece inúmeras vantagens na assistência prestada.

Neste contexto, é frequentemente sugerido que o papel dos enfermeiros se tornará cada vez mais importante na prestação futura de cuidados a $\mathrm{PcH}^{(5-10,21)}$. Segundo o European Haemophilia Consortium:

Os enfermeiros são um recurso extremamente valioso no atendimento de pacientes com hemofilia e assumem cada vez mais responsabilidades, incluindo tratamento de hemorragias agudas, organização de clínicas ambulatoriais, treinamento de pais e crianças em punção venosa e profilaxia, e prescrição de concentrados de fator de coagulação e outras medicações ${ }^{(27: 14)}$. 
O perfil das juízas que validaram o instrumento evidenciou um elevado número de atendimento mensal aos pacientes de coagulopatias, demonstrando experiência assistencial em hematologia. $O$ instrumento foi construído num hemocentro de referência de atendimento a $\mathrm{PcH}$ do Nordeste brasileiro, e validado por nove especialistas de hemocentros do país, propiciando maior confiabilidade e legitimidade ao instrumento.

A análise do instrumento pelas juízas demonstrou a necessidade de acrescentar dados e aperfeiçoar categorias para melhorar seu conteúdo, sendo inseridos: número de cigarros/ dia; presença de inibidor, data do último treinamento, primeiro título, pico histórico, título geral; treinamento de infusão/autoinfusão do fator de coagulação; imunização; número de filhos, atividade sexual, planejamento familiar e atividades esportivas.

A aplicação da CE requer o treinamento contínuo de enfermeiras para raciocínio clínico, usando ferramentas do exame clínico ${ }^{(4-6)}$ bem como para diagnóstico de enfermagem, de forma que foram acrescentados no instrumento os diagnósticos de enfermagem e os encaminhamentos realizados pelas enfermeiras de forma a garantir a autonomia.

A versão final do instrumento foi aplicada a uma amostra de $30 \mathrm{PcH}$, por meio de um teste piloto, e não houve dificuldade de aplicação pelas enfermeiras do ambulatório.

Segundo Ministério da Saúde, o enfermeiro precisa realizar três consultas por hora, não havendo distinções entre a consulta nova e a de seguimento ${ }^{(28)}$. Portanto, o enfermeiro dispõe em média de 20 minutos para realizar a consulta de enfermagem. Observou-se que o tempo de duração das consultas para aplicação do instrumento da pesquisa foi reduzido com a prática da aplicação do instrumento, atingindo a média preconizada.

Semelhantemente, estudo internacional(29) verificou duração média das CE de 10 minutos, com o mínimo de quatro minutos e o máximo de 35 minutos. Os autores afirmaram que a duração da consulta está associada às características do cliente, presença de comorbidades e do nível de prática do profissional. Nesta pesquisa, $\mathrm{PcH}$ apresentavam várias comorbidades como hemartrose, artropatias, inibidores contra fatores de coagulação, o que pode ter contribuído para um maior tempo na aplicação do instrumento nas primeiras consultas.

Verificou-se que a maioria das juízas não apresentou dificuldade em compreender o instrumento, fato positivo na avaliação, demonstrando que não houve diferenças significativas no uso do instrumento entre os grupos. $O$ instrumento foi implantado no serviço em formato eletrônico, e enfermeiros de outros hemocentros do Nordeste do país estão sendo treinados para seu uso.

A limitação do estudo consistiu na ausência de estudos sobre instrumento e/ ou protocolo de consulta de enfermagem para pessoa com hemofilia ou coagulopatias hereditárias, que servissem de modelo ou comparação dos resultados.

\section{CONCLUSÃO}

Elaborar e validar um instrumento de CE para hemofilia é importante para a prática clínica e científica da enfermagem, pois representa uma inovação na tomada de decisão, na aplicação de terminologias padronizadas, proporcionando autonomia, apoio técnico e respaldo ético ao enfermeiro. Entretanto, outros estudos são sugeridos: verificou-se lacuna bibliográfica sobre os instrumentos de CE para hemofilia, tanto nacional como internacionalmente. 
1. Srivastava A, Brewer AK, Mauser-Bunschoten EP, Key NS, Kitchen S, Llinas A, et al. Guidelines for the management of hemophilia. Haemophilia. [Internet]. 2013 [acesso em 04 jul 2019]; 19(1). Disponível em: https://doi.org/10.1111/j.1365-2516.2012.02909.x.

2. Scott MJ, Xiang H, Hart DP, Palmer B, Collins PW, Stephensen D, et al. Treatment regimens and outcomes in severe and moderate haemophilia A in the UK: the Thunder study. Haemophilia. [Internet]. 2019 [acesso em 04 jul 2019]; 25(2). Disponível em: http://doi.org/10.1111/hae.13616.

3. World Federation of Hemophilia. Annual Global Survey 2017 [Internet]. Montreal; 2018 [acesso em 08 ago 2019]. Disponível em: http://www1.wfh.org/publications/files/pdf-1714.pdf.

4. O'Shea E, Coughlan M, Corrigan H, McKee G. Evaluation of a nurse-led haemophilia counselling service. Br J Nurs. [Internet]. 2012 [acesso em 17 jul 2019]; 21(14). Disponível em: http://doi. org/10.12968/bjon.2012.21.14.864.

5. Khair K, Chaplin S. What is a nurse-led service? A discussion paper. J Haem Pract. [Internet]. 2017 [acesso em 04 jul 2019]; 4(1). Disponível em: http://doi.org/10.17225/jhp00100.

6. Khair K, Abu-Riash M, Acerbi AC, Beijlevelt M, Floros G, Li K, et al. Haemophilia nursing practice: a global survey of roles and responsibilities. J Haem Pract. [Internet]. 2016 [acesso em 20 jul 2019]; 3(2). Disponível em: http://doi.org/10.17225/jhp00078.

7. Polinski JM, Kowal MK, Gagnon M, Brennan TA, Shrank WH. Home infusion: safe, clinically effective, patient preferred, and cost saving. Healthc (Amst). [Internet]. 2017 [acesso em 08 jul 2019]; 5(1-2). Disponível em: http://doi.org/10.1016/j.hjdsi.2016.04.004.

8. Santaella ME, Bloomberg M, Anglade D. Home infusion teaching practices at federally funded hemophilia treatment centers in the United States of America. Res Pract Thromb Haemost. [Internet]. 2017 [acesso em 08 jul 2019]; 1(1). Disponível em: http://doi.org/10.1002/rth2.12020.

9. Lock J, Raat H, Peters M, Scholten M, Beijlevelt M, Oostenbrink R, et al. Optimization of home treatment in haemophilia: effects of transmural support by a haemophilia nurse on adherence and quality of life. Haemophilia. [Internet]. 2016 [acesso em 17 jul 2019]; 22(6). Disponível em: http://doi.org/10.1111/ hae.13043.

10. Schrijvers LH, Schuurmans MJ, Fischer K. Promoting self-management and adherence during prophylaxis: evidence-based recommendations for haemophilia professionals. Haemophilia. [Internet]. 2016 [acesso em 13 jun 2019]; 22(4). Disponível em: http://doi.org/10.1111/hae.12904.

11. Conselho Regional de Enfermagem de São Paulo. Guia para construção de protocolos assistenciais de Enfermagem [Internet]. São Paulo: COREN-SP; 2017 [acesso em 08 ago 2019]. Disponível em: http:// www.coren-sp.gov.br/sites/default/files/Protocolo-web.pdf.

12. Catunda HLO, Bernardo EBR, Vasconcelos CTM, Moura ERF, Pinheiro AKB, Aquino P de S. Percurso metodológico em pesquisas de enfermagem para construção e validação de protocolos. Texto contexto - enferm. [Internet]. 2017 [acesso em 05 jun 2018]; 26(2). Disponível em: http://dx.doi.org/10.1590/0104$\underline{07072017000650016 .}$.

13. Benson J, Clark F. A guide for instrument development and validation. Am J Occup Ther. [Internet]. 1982 [acesso em 21 maio 2017];36(12). Disponível em: http://dx.doi.org/10.5014/ajot.36.12.789.

14. Rezende SM, Rodrigues SHL, Brito KNP, Silva DLQ da, Santo ML, Simões B de J, et al. Evaluation of a web-based registry of inherited bleeding disorders: a descriptive study of the Brazilian experience with HEMOVIDAweb Coagulopatias. Orphanet J Rare Dis. [Internet]. 2017 [acesso em 15 maio 2018]; 12(27). Disponível em: https://ojrd.biomedcentral.com/articles/10.1186/s13023-016-0560-6. 
15. Massaroli A, Martini JG, Lino MM, Spenassato D, Massaroli R. The Delphi method as a methodological framework for research in nursing. Texto contexto-enferm. [Internet]. 2017 [acesso em 05 jun 2019]; 26(4). Disponível em: http://dx.doi.org/10.1590/0104-07072017001110017.

16. Arango HG. Bioestatística: teórica e computacional. 3. ed. Rio de Janeiro: Guanabara Koogan; 2009.

17. Polit DF, Beck CT. Fundamentos da pesquisa em enfermagem: avaliação de evidências na prática de enfermagem. 7. ed. Porto Alegre: Artmed; 2011

18. Voutillainen A, Pitkäaho T, Kvist T, Vehviläinen-Julkunen K. How to ask about patient satisfaction? The visual analogue scale is less vulnerable to confounding factors and ceiling effect than a symmetric Likert scale. J Adv Nurs. [Internet]. 2015 [acesso em 23 jun 2019]; 72(4). Disponível em: http://doi.org/10.1111/ jan.12875.

19. Coluci MZO, Alexandre NMC, Milani D. Construção de instrumentos de medida na área da saúde. Ciênc saúde coletiva. [Internet]. 2015 [acesso em 12 maio 2020]; 20(3). Disponível em: http://dx.doi. org/10.1590/1413-81232015203.04332013.

20. Vieira CENK, Enders BC, Coura AS, Menezes DJC de, Lira ALB de C, Medeiros CCM. Validación de instrumento para la detección de adolescentes con sobrepeso en la escuela. Enfermería Global. [Internet]. 2016 [acesso em 7 jul 2020]; 15(3). Disponível em: https://doi.org/10.6018/eglobal.15.3.221531.

21. Costa NC de M, Costa IM, Guimarães TMR, Souza HP de, Casaretto ES, Medeiros TC de, et al. North-eastern Brazilian recommendations for the nurse professional role on the pharmacokinetic-assisted prophylaxis individualization for haemophilia A. Haemophilia. [Internet]. 2020 [acesso em 02 nov 2020]; 26(3). Disponível em: https://pubmed.ncbi.nlm.nih.gov/32026514/.

22. Ferreira RC, Montanari FL, Ribeiro E, Correia MDL, Manzoli J, Duran ECM. Elaboração e validação de instrumento de assistência de enfermagem para pacientes em unidades de terapia intensiva. Cogitare enferm. [Internet]. 2018 [acesso em 24 set 2020]; 23(4). Disponível em: http://dx.doi.org/10.5380/ ce.v23i4.57539.

23. Alves MG, Pereira VOS, Batista DFG, Cordeiro ALP de C, Nascimento J da SG, Dalri MCB. Construção e validação de questionário para avaliação de conhecimento sobre ressuscitação cardiopulmonar. Cogitare enferm [Internet]. 2019 [acesso em 25 set 2020]; 24. Disponível em: http://dx.doi.org/10.5380/ ce.v24i0.64560.

24. Tolentino GS, Bettencourt AR de C, Fonseca SM da. Construção e validação de instrumento para consulta de enfermagem em quimioterapia ambulatorial. Rev. bras. enferm. [Internet]. 2019 [acesso em 26 set 2020]; 72(2). Disponível em: http://dx.doi.org/10.1590/0034-7167-2018-0031.

25. Moraes JT, Fonseca DF da, Mata LRF da, Oliveira PP de, Sampaio F de C, Silva JF da. Validation of a tool for nursing appointment to the person with diabetes mellitus and/or systemic hypertension. Rev. Enf. Ref. [Internet]. 2018 [acesso em 26 set 2020]; serIV(19). Disponível em: http://dx.doi.org/10.12707/ RIV18041.

26. Ministério da Saúde (BR). Secretaria de Atenção à Saúde. Departamento de Atenção Especializada e Temática. Manual de hemofilia. 2. ed. Brasília: Ministério da Saúde; 2015.

27. Berntorp E, Hart D, Mancuso ME, D'Oiron R, Perry D, O'Mahony B, et al. The first Team Haemophilia Education meeting, 2015, Amsterdam, The Netherlands. Eur J Haematol. [Internet]. 2016 [acesso em 05 jun 2020]; 97(suppl83). Disponível em: https://www.ncbi.nlm.nih.gov/pubmed/27272000.

28. Dantas CN, Santos VEP, Tourinho FSV. Nursing consultation as a technology for care in light of the thoughts of Bacon and Galimberti. Texto contexto - enferm [Internet]. 2016 [acesso em 26 set 2020]; 25(1). Disponível em: https://doi.org/10.1590/0104-0707201500002800014.

29. Stevens S, Bankhead C, Mukhtar T, Perera-Salazar R, Holt TA, Salisbury C, et al. Patient-level and practice-level factors associated with consultation duration: a cross-sectional analysis of over one million consultations in English primary care. BMJ Open [Internet]. 2017 [acesso em 10 out 2020]; 7(11).

Disponível em: https://bmjopen.bmj.com/content/7/11/e018261.long. 


\section{COMO REFERENCIAR ESTE ARTIGO:}

Andrade IAF de, Guimarães TMR, Costa IM, Costa NC de M, Camelo RM, Lima FM de. Construção e validação de instrumento de consulta de enfermagem para pessoas com hemofilia. Cogitare enferm. [Internet]. 2021 [acesso em "colocar data de acesso, dia, mês abreviado e ano"]; 26. Disponível em: http://dx.doi.org/10.5380/ ce.v26i0.74467.

Recebido em: 12/06/2020

Aprovado em: 13/11/2020

Editora associada: Luciana Alcântara Nogueira

\section{Autor Correspondente:}

Tânia Maria Rocha Guimarães

Universidade de Pernambuco - Recife, PE, Brasil

E-mail: tmrguimaraes@gmail.com

\section{Contribuição dos autores:}

Contribuições substanciais para a concepção ou desenho do estudo; ou a aquisição, análise ou interpretação de dados do estudo - TMRG, IMC, NCMC, FML

Elaboração e revisão crítica do conteúdo intelectual do estudo - RMC

Aprovação da versão final do estudo a ser publicado - TMRG, FML

Responsável por todos os aspectos do estudo, assegurando as questões de precisão ou integridade de qualquer parte do estudo - IAFA

Copyright $\odot 2021$ Este é um artigo em acesso aberto distribuído nos termos da Licença Creative Commons Atribuição, que permite o uso irrestrito, a distribuição e reprodução em qualquer meio desde que o artigo original seja devidamente citado. 